Western University

Scholarship@Western

Psychology

Psychology

1989

\title{
Categorical Modelling of Husserl's Intentionality
}

Imants Barušs

King's University College, baruss@uwo.ca

Follow this and additional works at: https://ir.lib.uwo.ca/kingspsychologypub

Part of the Psychology Commons

Citation of this paper:

Barušs, Imants, "Categorical Modelling of Husserl's Intentionality" (1989). Psychology. 19.

https://ir.lib.uwo.ca/kingspsychologypub/19 


\section{CATEGORICAL MODELLING OF HUSSERL'S INTENTIONALITY}

Imants Barus s

King's College, The University of Western Ontario

This paper is concerned with the application of constructions from category theory to Smith and McIntyre's interpretation of Husserl's intentionality. ${ }^{1}$ Not only did Husserl's own ideas change in the course of his lifetime ${ }^{2}$ but there are a number of interpretations of Husserl's work ${ }^{3}$ so that the line of philosophical investigation that Husserl strongly influenced is still in the process of development. In this vein, Smith and McIntyre have recognized the potential for a possible worlds interpretation of intentionality in Husserl's writing ${ }^{4}$ which has led them to extend their interpretation to give a many worlds account of intentionality. ${ }^{5}$ Thus, while Smith and McIntyre have refuted interpretations of Husserl's noema in terms of ideal objects, they have been willing to explicate the noema using possible objects. ${ }^{6}$

There are, however, limitations to present possible worlds theories. Most notable, perhaps, is the static nature of such worlds. By isolating a discrete act of consciousness, one constructs a collection of possible worlds that belong to that act. What one does, thus, for a single act is analogous to what one does when interpreting an elementary classical theory using classical models.

The motivation for providing a possible worlds account of intentionality, however, arises from the implicit presence of collections of acts which, as it were, further define an initial act in a number of possible, possibly incompatible, ways. One would like to be able to take into account the presence of such acts along the dimension of time which, naturally, locally, orders such acts. In an effort to do this one can seek inspiration from some of the developments in intuitionistic logic which have incorporated the notion of movement ${ }^{7}$ and more specifically, from the Kripke-Joyal semantics which explicitly involve categorical constructions. ${ }^{8}$ The modelling given here is not, of course, a semantics for a formal 
language, but an interpretation directly of mental events, in terms of categorical constructions used for the Kripke-Joyal semantics.

In Section 1 the key elements of Smith and McIntyre's interpretation of Husserl's intentionality are briefly sketched. Specific categorical constructions are developed in Section 2 and shown to correspond to the elements of an intentional mental event in Section 3. Some specific issues regarding the interpretation of the past, beliefs and actuality are raised in Section 4. In Section 5 the discussion is concluded by comparing the categorical interpretation, developed in Sections 3 and 4, with the many worlds interpretation of Smith and McIntyre.

\section{HUSSERL'S INTENTIONALITY.}

Husserl was interested in explaining the nature of those mental events which are characterized by intentionality. He called 'acts of consciousness', those events which consist in "( $a$ conscious subject's) intending... or being directed... to, or being related... to, an object." Instead of explicating intentionality by examining the nature of the objects towards which acts of consciousness are directed, Smith and McIntyre have claimed that Husserl's account can be viewed as one which seeks to understand intentionality in terms of the relationship between the subject and the object. ${ }^{10}$

Each conscious act, according to Smith and McIntyre, consists of three components a noesis, a noema and an object. The noesis is the real part of a conscious act which makes the experience intentional. ${ }^{11}$ The noema, on the other hand, is not 'real' but an atemporal kind of meaning or Sinn. ${ }^{12}$ The ontological status of the object is unimportant on this account. Thus, "an act intends (is directed toward or is intentionally related to) an object if and only if the act (or its noesis) entertains a certain noematic Sinn and that Sinn prescribes that object." 13

Objects of natural experience are transcendent objects in the sense that they can 
never be fully conceptually captured in a single act. This is due to the fact that the perceptual evidence for the existence of such objects is always incomplete from the point of view of a single act. ${ }^{14}$ In fact, perceptual experiences are more complicated than other intentional experiences such as, for example, judging, remembering and imagining, because they entail a sensory aspect. The barrage of sensations or hyle is an aspect of the noesis and requires a Sinn to give it shape so that it can be experienced as a perception of something. ${ }^{15}$ For Husserl, perception forms the basis of one's understanding of the world and hence received much of his attention. ${ }^{16}$

This problem of the perception of transcendent objects highlights the fact that no act of consciousness exists in isolation from other acts. Something about the object is always left open in an act with a particular Sinn. In order to explicate the way in which further determinations of an object are possible, Husserl introduced the notion of an act-horizon a collection of possible acts whose Sinne are compatible with the Sinn of the original act, that can further determine a given object. ${ }^{17}$ Parallel to this act-horizon is an object-horizon which is the collection of further determined objects that these further acts. of consciousness are directed towards. ${ }^{18}$

According to Smith and McIntyre's interpretation of Husserl's theories, each Sinn can be broken down into two components: the 'predicate senses' which prescribe the properties that an object is to have, and an $X$ which specifies the object to which the properties are ascribed. ${ }^{19}$ Further determination of an object, then, consists of further conditions that are to be met by an object, given in acts with the same $X$.

Following an examination of Husserl's notions of horizon and manifold, however, Smith and McIntyre relaxed the constraints on the definition of an act-horizon, so that the Sinne of the acts in the horizon need not be strictly compatible with the Sinn of the original act, ${ }^{20}$ allowing for further perceptions of an object that reveal it to be different from what it was originally perceived to be, and so that these further acts need not 
necessarily further determine the object in the sense of including further information about it. $^{21}$ It is this more liberal interpretation of horizon that is adopted here.

For Husserl, each act-horizon is predelineated in an act of consciousness so that some possible acts are understood to further determine the object of the original act, and others are seen as not doing so. These further possible acts cannot be merely logically possible, but must be motivated by what the subject believes to be possible. ${ }^{22}$ This brings to light the importance of relevant background beliefs and items of knowledge that determine which Sinne are to be included in an act-horizon. ${ }^{23}$

The act-horizon has a temporal structure. In the case of perception, for example, one can have a series of possible future perceptions that further determines an object. Thus, if one were to see a tree on a hillside, walking closer to the tree may reveal it to be a maple tree with red leaves, and so on. These further possible perceptions would be acts in the horizon of the original act in which the tree is first noticed. Now, if the tree were a maple tree with red leaves on a hillside, then there would be a number of possible combinations of further perceptions that would reveal this. Smith and McIntyre call continuous series of possible further perceptions, 'possible verification chains', and collections of series of further perceptions, which reveal the same object, they call a family of possible verification chains. ${ }^{24}$ Thus, the act-horizon can be articulated by talking about the collection of all families of possible verification chains.

The introduction of families of verification chains allows for the development of a possible worlds interpretation of Husserl's intentionality. 'Each family of verification chains corresponds to a possible object, but since these acts which constitute the horizon are to be co-directed with the original act, that original act can be understood to be directed to any of a number of possible objects in possible worlds.

Co-directedness requires that the Sinne of any two acts in the horizon have the same determinable $X$ - the same $X$ as the Sinn of the original act - and 
that their respective "contents" (conjoint predicate--senses) be mutually

"harmonious", compatible according to the subject's conceptual scheme. ${ }^{25}$

It is this ability to identify a number of different ways of completing the same object intended in the act, by examining that act's horizon, that allows for a possible worlds account of intentionality.

However, Smith and McIntyre, in their extension of Husserl's work, forsake the very temporal structure that allows for the presence of possible objects by conceiving of them as possible objects in possible worlds reached by a choice function for any given act. The effort here, on the other hand, is to retain the temporal structure of the horizon and to show how specific categorical constructions, those used in the definition of Grothendieck topoi, can provide a more dynamic many worlds interpretation of intentionality.

\section{GROTHENDIECK TOPOI.}

Some simple constructions from category theory are introduced in this section, with the assumption that the reader is familiar with the basic notions of set theory. Categories, pretopologies, sites, functors and sheaves, the ingredients of Grothendieck topoi, are defined below. ${ }^{26}$ These will be used to model Husserl's intentionality in the remainder of the paper.

To begin with, a category is a collection of nodes with arrows between them, known as a directed graph, to which further conditions apply. The nodes are usually called objects and the arrows sometimes also called morphisms. If $C$ is à category, then $C O b^{27}$ is the collection of objects of $C$ and $C A \mathrm{r}^{28}$ is the collection of arrows of $C$. The object at the tail of an arrow is known as the domain and that at the head of an arrow as the codomain. If $C$ is a category and $f$ is an arrow of $C$, with domain $a$ and codomain $b$, then this arrow is usually denoted as $a \stackrel{f}{f_{b}}$.

In order for a directed graph to be a category, it must obey the laws of closure, 
identity and associativity:

(i) Closure: If $a \stackrel{f_{4}}{b}$ and $b \stackrel{g}{\longrightarrow} c$ are arrows of a category $C$, then there exists an arrow in $C$ which consists of $f$ followed by $g$. This arrow is known as the composition of $f$ and $g$ and is designated by $a \stackrel{f g}{ } c$.

(ii) Identity: For each object $a$ of a category $C$, there is an identity arrow, $1_{a}$, with the property that, if $a \stackrel{L_{b}}{b}$ is an arrow of $C$, then $1_{a} f=f=f 1_{b}$.

(iii) Associativity: If $a \stackrel{f_{b}}{\longrightarrow} \stackrel{g}{\longrightarrow}_{c} \stackrel{h}{\longrightarrow} d$ is a configuration of objects and arrows of $C$, then $f(g h)=(f g) h$.

Thus, a category is a collection of objects and arrows with some straightforward properties. The collection of all sets with functions between them is the most obvious example of a category.

Different pathways in a category can be indicated using diagrams. If all the pathways between any two objects in a diagram are the same arrow, then the diagram is said to commute. For example, the following configuration of objects and arrows from a category $C$

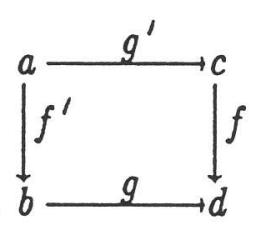

is a diagram. If $f^{\prime} g=g^{\prime} f$ then the diagram commutes.

Furthermore, the above diagram is a pullback square if it has the universal property that for every $x \in C O b$ and arrows $x \stackrel{\emptyset}{\longrightarrow} b$ and $x \stackrel{\psi}{\longrightarrow}$ c with $\emptyset g=\psi f$, there exists a unique arrow $x \stackrel{\sigma}{\longrightarrow} a$ that makes the following diagram commute.

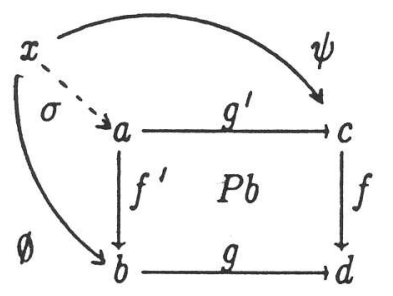


The $P b$ inside a square identifies it as a pullback square.

With the aid of the notion of a pullback square, the concept of locality can be introduced in a category. This is done by associating with each object in a category a number of collections of arrows that terminate at that object. Such collections of arrows, satisfying the closure conditions below, are called covers. If an object that is the domain of an arrow is thought of as a 'future' state with respect to the codomain of an arrow, then different covers can be interpreted as different possible futures.

More formally, a pretopology is an assignment ${ }^{29}$

$$
\text { Cov : } \mathrm{COb} \longrightarrow(C A r) P P
$$

which takes $a \in C O b$ to a collection of sets of arrows of $C$ with codomain $a$, satisfying the following conditions:

(i) $\emptyset \notin a \operatorname{Cov}$

That is to say, the empty set cannot be a cover of $a .^{30}$

(ii) $\{a \stackrel{1}{a}, a\} \in a$ Cov

That is to say, the singleton consisting of the identity will always be a cover of an object.

(iii) Let $X$ be an index set, and $Y_{x}$ be index sets for $x \in X$. If $\left\{a_{x} \stackrel{f_{x}}{\longrightarrow} a: x \in X\right\} \in a$ Cov and for each $x \in X,\left\{a_{y}^{x} \stackrel{f}{y}_{x}: y \in Y_{x}\right\} \in a_{x} \operatorname{Cov}$ then $\left\{a \stackrel{x f_{y}^{x} f_{x}}{\longrightarrow} a: y \in Y_{x}\right.$ and $\left.x \in X\right\} \in a$ Cov.

In other words, covers can extend indefinitely into the future, since the elements of the covers of the codomains of arrows in a cover of a can themselves be composed with the elements of a cover of $a$ to give a new cover.

(iv) If $\left\{a \frac{f_{x}}{\longrightarrow} a: x \in X\right\} \in a$ Cov, where $X$ is an index set, and $b \stackrel{g}{\rightarrow} a \in C A r$, then for each $x \in X$ the pullback $b \times{ }_{a} a_{x} \stackrel{f_{x}^{\prime}}{\longrightarrow} b$ of $f_{x}$ along $g$ 


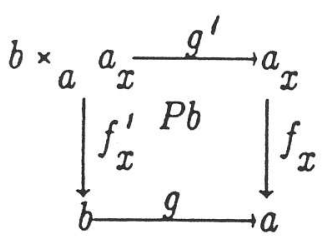

exists and $\left\{b \times{ }_{a} a_{x} \stackrel{f_{x}^{\prime}}{\longrightarrow} b: x \in X\right\} \in b$ Cov. In other words, for an assignment to be a pretopology, any specific cover must be sufficiently mobile to become a cover of any possible future state.

A category $C$, together with a pretopology $\operatorname{Cov}$, as an ordered pair $\langle C$, Cov $\rangle$, constitute a site. A site can be thought of as a temporal domain in which certain specific futures can be associated with each other. The next step is to identify the categorical structures which can be used for the interpretation of events that take place within this temporal grid. To do this, the notion of a functor is needed.

A functor is a structure-preserving function between categories. As such, it takes objects to objects, arrows to arrows, and preserves composition and identity. That is to say, for categories $C$ and $D$, a functor $F$ is defined as a function $F: C \longrightarrow D$ with action $a \longmapsto a F$ for objects and $\left(a \stackrel{L_{b}}{b) \longmapsto}(a F \stackrel{f F}{\longrightarrow} b F)\right.$ for arrows so that

(i) $(f g) F=f F g F$ whenever $f g$ is defined ${ }^{31}$

(ii) $1_{a} F=1_{a F}$ for all $a \in C O b$.

A contravariant functor is a functor that turns around the directions of arrows so that domains become codomains, and codomains become domains. That is to say, in the case of a contravariant functor, the action for arrows becomes

$$
(a \stackrel{f}{L} b) \longmapsto\left(b F \stackrel{f F}{\longmapsto} a F^{\prime}\right)
$$

and (i) above becomes

$$
(f g) F=g F f F \text { whenever } f g \text { is defined in } C A r^{32}
$$

The functors of interest here are contravariant functors that map arbitrary categories into the category $S$ whose objects are sets and whose arrows are functions. The functor itself is 
called a stack. For $a \in C O b, a F$ is called a stalk or fibre over $a$, and $s_{a} \in a F$ is called a germ.

This construction can be visualized as a planar grid made up of the objects and arrows of a category with stalks, filled with germs, sitting over the objects like wheat in a wheat field. What happens in the course of modelling, is that the categorical grid represents temporal movement, the stalks the directedness at a given moment, and the germs the particulars of events. All that remains now is to employ the notion of locality as given by the pretopologies.

Let $\left\langle C\right.$, Cov $>$ be a site and $\left\{a \stackrel{f_{x}}{\longrightarrow} a: x \in X\right\} \in a$ Cov then the pullback of $f_{x}$ along $f_{y}$ is called $a_{x}{ }^{\times} a_{a} a_{y} \stackrel{f_{x}^{\prime}}{\longrightarrow} a_{y}$ and the pullback of $f_{y}$ along $f_{x}$ is called $a_{x}{ }^{\times}{ }_{a} a_{y} \stackrel{f_{y}^{\prime}}{{ }_{a}} a_{x}$ for each $x$ and $y$ in the index set $X$, as shown in the diagram.

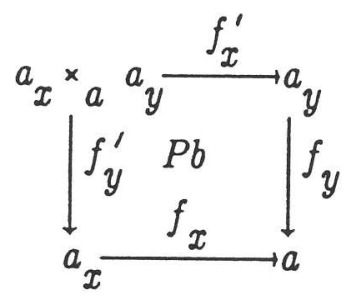

If $F$ is a stack over $C$ then the image of $a_{x}{ }^{\times} a_{a} a_{y} \stackrel{f_{y}^{\prime}}{a_{x}}$ is called $a_{x} F \stackrel{F_{y}^{x}}{\longrightarrow}\left(a_{x}{ }^{\times} a_{a} a_{y}\right) F$ and the image of $a_{x} \stackrel{f_{x}}{\longrightarrow} a$ is called $a F \stackrel{F_{x}}{\longrightarrow} a_{x} F$, for all $x, y \in X$.

The germs in the stalks are not unrelated to one another, but follow in reverse direction, the pathways taken by the arrows. Thus, for $s_{a} \in a \dot{F}$, if $b \stackrel{g}{\rightarrow} a \in C A r$, then $s_{a}$ gets carried to $s_{a} g F \in b F^{33}$. Now, the pretopology on a category can be used to define characteristics of the relationships between the germs. In particular, if germs in stalks over a cover coinciding in future states given by pullbacks originated from the same germ in the present, then this gives a way of characterizing stalks for which future conditions give information about a current state of affairs.

More formally, a stack $F$ is a sheaf over the site $\langle C$, Cov $\rangle$ if it satisfies the 
Canporability Condition

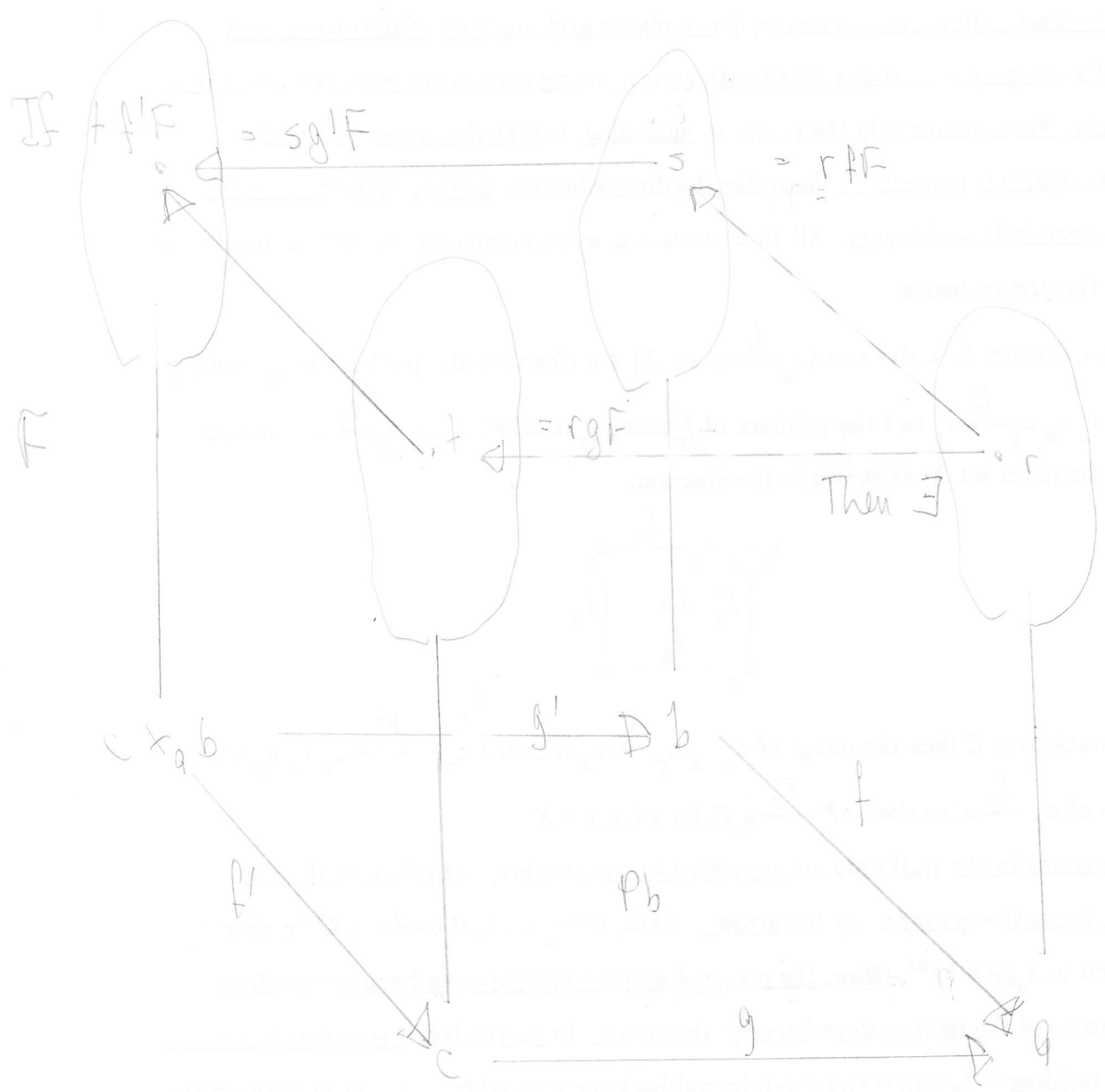


compatibility condition:

Given any cover $\left\{a \frac{f_{x}}{\longrightarrow} a: x \in X\right\} \in a$ Cov for $a \in C O b$ and any collection of germs $\left\{s_{x} \in a_{x} F: x \in X\right\}$ that are pairwise compatible, i.e., $s_{x} F_{y}^{x}=s_{y} F_{x}^{y}$ for all $x, y \in X$, there is exactly one $s_{a} \in a F$ so that $s_{a} F_{x}=a_{x}$ for all $x \in X$.

That is to say, whenever the germs of stalks in a cover that are pairwise pulled back and shown to be the same originated from a single germ, then the stack is known as a sheaf.

The collections of sheaves over a site generate a category C Sh called a Grothendieck topos.

\section{A CORRESPONDENCE BETWEEN INTENTIONALITY AND TOPOI.}

The correspondence between acts of consciousness and the elements of Grothendieck topoi hinges on the use of a category to reflect the organization of possible acts in time, the identification of germs with the possible objects of mental events characterized by intentionality and of a cover of a categorical object with a family of verification chains. It is the purpose of this section to explicate these ideas.

A starting point for the development of this modelling, is the noticing that lived acts of consciousness can be thought of as discrete events, strung together, somehow, in time, as part of the experience of a person. ${ }^{34}$ Thus an object $a$ in a category $C$ can be thought of as a single act, with the arrows between objects as the transitions from one possible act to another. For the purposes of this modelling, a future event for event $a$ would be an event $b$ for which there is an arrow $b \stackrel{g}{g} \in \in A$ Ar. The direction for the arrow can be interpreted by thinking of future events as those coming towards a person. If one allows only future acts that cannot be genuine replications of past acts, one could choose only categories in which there are no arrows $a \stackrel{h}{\longrightarrow} b \in C$ Ar for every future $b^{35}$ If one makes the further restriction that a person can get from one act to a next in only one way, then the category representing possible acts in time becomes a partially ordered set. Leaving open the widest 
scope for interpretation, in this discussion, categories, as such, correspond to the domain of possible acts of consciousness. To each person one assigns a category that represents their possible past, present and future acts. Within such a category, a person would trace a path from the past into the future.

Each intentional, mental event, for Husserl is characterized by a Sinn or meaning that directs the act towards an object. As Smith and McIntyre have suggested, the burden carried by the Sinne can be moved to the objects that an act is directed towards, where those objects are possible objects. ${ }^{36}$ In the spirit of their enterprise, the Sinn is here dissected so that the directedness itself is modelled by the sheaves which determine the stalks over the objects in a category, the predicate contents and $X$ replaced by the possible objects which are modelled by the germs in the stalks, and any residual experience of meaning that may characterize noemata, moved into the categorical object itself, which is, basically, the noesis, the lived part of the act. ${ }^{37}$ Thus, the directedness of an act is here considered to be the action of a single sheaf $F$ in $C$ Sh on an object $a$ of $C$.

It is important to notice that possible objects persist in time. For example, if an act at $a$ is directed towards the germ $s_{a} \in a F$, then, for any future state $b$ with $b \stackrel{g}{\longrightarrow} a \in C A r$, the object $s_{a}$ of the mental act $a$ becomes the possible object $s_{a} g F \in b F$ by the action of the function $a F \stackrel{g F}{\longrightarrow} b F \in S$ Ar. Because of the contravariance of the functor $F$, objects that appear at a given point in time, are carried, possibly in changed form, into the future.

The interesting feature of Husserl's intentionality however, that sheaves over a site allow one to model, is not the simple structure of intentional mental events and their effects on the future, but the influence of the future on the present. In order to model this, the families of possible verification chains, the collections of temporal sequences that determine possible objects, are to correspond to the covers of an object $a$ in a category $C$. This allows for one way of making precise the notions of synthesis of identification, whereby the Sinne of the acts in an act-horizon form one 'composite Sinn' and the identity 
of the $X$ across the acts in an act-horizon.

In the first case, by conceptualizing an act horizon as a collection of verification chains, themselves consisting of possible, though experiencable acts, one replaces the synthesis of identification of the Sinne with the actual Sinne of the acts forming a cover. In the second case, the possible object determined by the Sinne of a verification chain would be more or less characterized in mutually compatible ways by the acts of a verification chain. Therefore, rather than retaining the notion of the object as static along the verification chain, one can allow it to be the object that would be determined in a particular act in an àct-horizon.

It may be useful to look at an example. One may see what looks like a tree in an eriginal act of perception. The act-horizon consists of a number of verification chains where this tree is explored in particular ways, revealing it to be perhaps a maple tree with red leaves, on a hillside, and so on. An alternate collection of verification chains may reveal the tree to be the holographic image of a maple tree with red leaves, on a hillside and so on. At the point of the initial act, one has not yet determined that the tree is a real tree rather than the holographic image of such a tree. There are at least three possible objects, then, that are present in the initial act for this example: the tree as it is determined without judgement as to its reality or non-reality, the tree interpreted as a maple tree and the tree interpreted as a holographic image of a maple tree. In other words, each object can be understood as a possible object, determined as that possible object only up to a certain point. That is to say, the changes of the object on a cover can be understood as completing, or verifying the possible object given in the initial act.

In such a formulation, the role that the object-horizon can have in determining the object in the initial act, is revealed by the compatibility condition. If there is a sequence of future mental events in which the objects that are determined are pairwise revealed to be the same object in appropriate further future states, then they can be said to determine a 
possible object in the original act. This is just a statement of the compatibility condition when the future mental states are the categorical objects of a cover for an object of an act of consciousness.

Finally, it can be shown that the collections of families of possible verification chains satisfy the defining conditions for a pretopology defined in the last section. Condition (i) is met trivially, in that an act-horizon must consist of at least one act. Condition (ii) is more interesting. It translates into the statement that an initial act must be an act-horizon in and of itself. This corresponds to the case in the example above where one suspends judgement as to whether or not the tree is a real tree or a holographic image and the object of the act is only that which is determined in the act itself.

Condition (iii) makes precise the notion that no object is ever fully determined, but subject to further determinations. Any act in the horizon is itself an original act with its collection of act-horizons. These help to determine the object of such an act in the act horizon, which in turn is part of the horizon of the original act.

Condition (iv) states that the possible determinations of objects at a given point in time are not lost, but modified under changes of circumstance. In the example above, suppose $b \stackrel{g}{a}$ is the action of turning around and speaking to someone and then facing the tree again. The options that one had initially are still there, but in modified form. Suppose that one had spoken to the artist responsible for the holographic maple tree. One carries additional information into act $b$ when one again confronts the tree. One is still left with the act-horizons that one started with, but the further acts would be modified because of the changes in expectation, perhaps, of what this object that is to be encountered is like. In act $b$, just as in act a however, the object need not be further determined, as demonstrated, for example, by considering that the person claiming to be the artist had lied about the tree being a holographic image.

Suppose, however, that the act $a \stackrel{g}{\longrightarrow} b$ were to take one into a very different situation. 
Suppose, for example, that the tree were to be vaporized in a nuclear holocaust and the observer, somehow, miraculously, spared, so that the original further determinations would not be possible. In such a case, the pullback of $g$, given as $\left(a \times{ }_{a} b\right) \stackrel{g^{\prime}}{a} a_{x}$ would give rise to a function $a_{x} F \stackrel{g^{\prime} F}{\longrightarrow}\left(a_{x} \times{ }_{a}{ }^{b}\right) F$ that would correctly reflect what had happened to $s_{x} \in a_{x} F,{ }^{38}$ namely, that it had been vapourized. In other words, at $b$ one would look at the possibility of verifying the presence of a vapourized tree or vapourized holographic image of a tree. One would never be able to decide, perhaps, which it would have been in the first place.

One of the benefits of this modelling, is that categorical structures allow for an interpretation in which objects are allowed to change faithfully with changes from one act to the next.

\section{THE PAST, BELIEFS AND ACTUALITY.}

For Husserl, an act's temporal horizon extends not only into the future, but into the past as well. There are, for example, possible past perceptions that one could have had, but did not have. ${ }^{39}$ These are easily modelled by considering arrows $a \stackrel{h}{\longrightarrow} c$ for a present act $a$ and possible, not experienced act $c$, that can nonetheless influence the experience at a. Similarly, the past portions of the verification chains of Smith and McIntyre ${ }^{40}$ can be seen as arrows leaving the present.

However, what can or cannot be recovered from the past is not a matter of the past, but of the present and the future - that is to say, the past is only applicable to an act insofar as it conditions what happens in the present or can be recovered in a further act In the present modelling, then, all of the possible pasts converge in the present which alone is necessary for understanding the future possibilities. This reflects the notion that the past has been completed by taking a specific path. The possibilities that could have existed and those that can exist in the future are a function of the beliefs that are possible at any given 
act. These beliefs themselves, are determined by the actual path that has been traversed. In keeping with the notion that beliefs predelineate the possible future determinations of an object, one has to posit that the pretopology Cov on a category $C$ is such that every cover specifies only those futures that are held to be possible. For example, if one is confronted with what appears to be a maple tree with red leaves and has no understanding of what a holographic image is, there is no cover for those future events which in fact reveal the maple tree with red leaves to be a holographic image. It is important to note here, that if, in fact, one is exposed to a holographic image, rather than a real tree, there are future events to substantiate that. These do not, however, constitute verification chains, since there is no possible object in the original act which is to be verified. In the absence of a cover, the compatibility condition does not apply, and despite the presence of compatible future objects, no possible object of the tree as a holographic image is present in the original act.

But this brings up the problem of actuality. Not all possible past or future paths can be traversed. Suppose one does, in the above example, attempt to traverse the future path that corresponds to the tree being a real tree, only to find out that it is a holographic image. In such a case, the continuous perception of the tree as a real tree explodes forcing the object one has chosen to be 'cancelled'. ${ }^{41}$ The hyle stemming from the actual world are not those of the original choice of object, but of an object which was not before present, though, or course, an unjudged portion of it could have been chosen as the object of the original act. It is interesting, however, to note that there is an interplay between the actual and the possible according to Husserl, because of the role that Sinne play in giving shape to sense impressions.

5. COMPARISON OF THE CATEGORICAL AND POSSIBLE WORLDS MODELS. In order to develop their possible worlds theory, Smith and McIntyre introduced the notion 
of possible verification chains, collections of which constitute an act's horizon, then abstracted the possible objects defined by families of such chains from the temporal setting in which they originate. Taking their cue from Carnap's meaning functions, they went on to define the Sinn as that function which selects, for any given possible world, an object in that world to be the object of an act. Smith and McIntyre made the distinction between a Husserlian possible worlds theory in which the notion of Sinn is retained, that is to say, a theory in which "an act's intentionality consists in a pattern of directedness that reaches into various different possible worlds under the noematic guidance of the act's Sinn"42 and a pure possible worlds theory in which the Sinn is deleted and which "assumes that intention consists in a complex relation, a pattern of directedness, that obtains between a person in a given possible world and certain possible objects, i.e., objects in various possible worlds". ${ }^{43}$

Besides the fact that these possible worlds have been isolated from the sequence of possible acts that gave rise to them, the pure possible worlds account appears to change the original structure of an act of consciousness in a fundamental way. As stated in the second quotation in the paragraph above, this account assigns to a person in a given possible world a possible object, thereby losing the self of intentional mental events. There is a difference between saying that the domain of a meaning function is a thinker over time or a collection of possible worlds. In the former case, the function replaces the directedness of intentionality. In the second case, the meaning function relates possible worlds and objects in those worlds without any reference to a self that persists between possible worlds.

One can, in fact, recover Smith and McIntyre's pure possible worlds theory of intentionality from the categorical account without losing the temporal context or the self. To do this, one lets the category $C$ be a partially ordered set ${ }^{44}$ and one defines a partial section to be a partial function from the elements of the partially ordered set $C O b$ to the collection of stalks over $C$ defined by a sheaf $F$, so that functoriality is preserved. That is 
to say, one defines a partial function $s: C O b u S O b$ with action $a \longmapsto s_{a}$ which is such that,

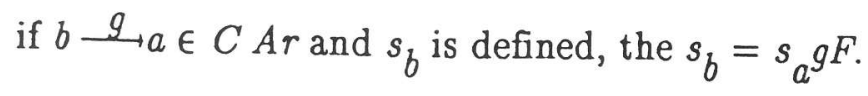

Husserl speaks of both an internal horizon and an external horizon, the former corresponding to the further determinations of the characteristics of an object, and the latter to the relationships that an object has to all other objects in its environment. 45 It is via the external horizon that, in principle, an entire possible world is revealed, in which a possible object is situated. But then, more generally, the complete future determinations of a germ $s_{a}$ at a reveal an entire possible world, the partial section $s$. Thus, the possible worlds of Smith and McIntyre are partial sections that are relevant to all acts, revealing how these possible worlds can develop, and are not just relevant to a single act. It is the category of possible acts itself, that glues together the determinations that form a possible world. Thus, the pattern of directedness of the pure possible worlds account is captured in the categorical account by the collection of partial sections over the partially ordered set of all acts of consciousness.

Another way of understanding possible worlds in the categorical context, superimposed on the first, is to consider different sheaves as radically different meanings that could be assigned to the same acts. Thus, instead of considering a single sheaf $F$, one could consider other sheaves in $C S h$ which would give perhaps entirely different collections of possible objects. Movement between sheaves would be via natural transformations, the arrows of $C S h$ that would continue to preserve the temporal dynamic. With the potential for the readmission of a number of sheaves in the modelling, the possibility of exploiting the topos structure of $C S h$ should be explored. The utilization of the subobject classifier for the assignemnt of truth-values to some of the arrows of C Sh may be a useful strategy.

In summary then, the modelling presented here, using categorical constructions, reveals the dynamics of Husserl's intentionality in a more explicit manner than does the possible worlds account of Smith and McIntyre. ${ }^{46}$ 
1. Smith and McIntyre's version of Husserl is based on D.W. Smith and R. McIntyre, Husserl and Intentionality - A Study of Mind, Meaning, and Language (Dordrecht, Holland: D. Reidel Publishing Company, 1982).

2. For example, Husserl's notion of Sinn or 'meaning' underwent changes, op. cit. p. 124.

3. For example, those given in H.L. Dreyfus, ed., Husserl, Intentionality, and Cognitive Science (Cambridge, Mass.: The MIT Press, 1982) or that of J.N. Mohanty, Edmund Husserl's Theory of Meaning (2nd ed.) (The Hague: Martinus Nijhoff, 1969).

4. Smith and McIntyre, op. cit., Chapter VI.

5. Smith and McIntyre, op. cit., Chapter VII.

6. Smith and McIntyre, op, cit., pp. 315-316.

7. For example, the work of A. Grzegorczyk "A Philosophically Plausible Formal Interpretation of Intuitionistic Logic", Indagationes Mathematicae, 26(1964), 596-601 and S. Kripke, "Semantical Analysis of Intuitionistic Logic I", in J.N. Crossley and M.A.E. Dummett, eds., Formal Systems and Recursive Functions-Proceedings of the Eighth Logic Colloquium, Oxford, July 1963 (Amsterdam: North-Holland Publishing Company, 1965).

8. Versions of the Kripke-Joyal semantics have been given by R. Goldblatt, Topoi-The Categorial Analysis of Logic, Volume 98 of Studies in Logic and the Foundations of Mathematics, (Amsterdam: North-Holland Publishing Company, 1979), and by A. Kock and G.E. Reyes, "Doctrines in Categorical Logic", in J. Barwise, ed., Handbook of Mathematical Logic (Amsterdam: North-Holland Publishing Company, 1977).

9. Smith and McIntyre op. cit., p. 5.

10. Ibid, Chapters II and III.

11. Ibid, p. 120.

12. Ibid, p. 121. 
13. Ibid, p. 143.

14. Ibid, p. 16.

15. Ibid, pp. 136-138.

16. Ibid, p. 241.

17. Ibid, p. 227.

18. Ibid, p. 231.

19. Ibid, p. 195.

20. That is to say, the predicate senses need not be compatible.

21. Ibid, p. 246.

22. Ibid, pp. 246-248.

23. Ibid, p. 249 and p. 254.

24. Ibid, pp. 259-260.

25. Ibid, p. 261. In practice, strict compatibility only applies to families of verification chains characterizing the same further determinations of an object. As noted previously, in the horizon, as such, counterfactual determinations are allowed.

26. The material in this section is principally based on J. Lambek and P.J. Scott, Introduction to Higher Order Categorical Logic_Cambridge Studies in Advanced Mathematics 7 (Cambridge: Cambridge University Press, 1986); Goldblatt, op. cit.; M.A. Arbib and E.G. Manes, Arrows, Structures, and Functors-The Categorical Imperative (New York: Academic Press, 1975); and Kock and Reyes, op. cit.

27. In this paper, composition of arrows, functions and functors is written using forward compositional notation. While this may seem awkward to those familiar with the usual backward notation of set theory, it makes categorical constructions easier to read.

28. For the purposes of the present paper, all references to categories are to small categories, that is to say, to categories in which the collection of arrows between any 
two objects is a set rather than a proper class.

29. In this expression, $(C A r) P P$ is the power set of the power set of arrows of $C$.

30. This first condition is not strictly necessary for the definition of a pretopology, and both Goldblatt, op. cit. and Kock and Reyes, op. cit. leave it out of their definitions of pretopologies. It is, however, necessary for the development of a semantics for a formal language, as well as for the present modelling.

31. Brackets are omitted from compositions that are unambiguous.

32. The function Cov can be extended to a contravariant functor from $C$ to the category of sets by assigning to the arrow $b \stackrel{g}{\rightarrow}$ of $C$ the function that takes a cover of $a$ to its corresponding cover on $b$ by (iv) of the above definition for a pretopology.

33. That is to say, $s_{a}\left(g F^{\prime}\right) \in b F$. Since a germ $s_{a}$ in a stalk $a F$ can be identified with the arrow $1 \stackrel{s_{a}}{\rightarrow} a F$ that takes the single element of the terminal object 1 into the element $s_{a}$ of $a F, s_{a} g F$ is a composition of arrows. Again, brackets are omitted whenever the composition of arrows and functors is uniquely determined.

34. One could argue that this is a degenerate characterization of time, as does, for example, M. Heidegger, Being and Time, trans. J. Macquarrie and E. Robinson (New York: Harper and Row, 1962).

35. One may wish to retain more general categories for a number of reasons. For example, in psychopathological cases in which there are conscious acts but no consolidation from short-term into long-term memory, one could identify two separate conscious acts in which virtually identical stimulus situations are experienced in the same way.

36. Smith and McIntyre op. cit., pp. 315-316.

37. Under such an interpretation, of course, the object does not, in a mathematical sense, bestow intentionality on the act as this may suggest. 
38. It is assumed here that $a \frac{f_{x}}{\longrightarrow} a$ is a transition to a future act in one of the verification chains for the original act.

39. Smith and McIntyre op. cit., p. 259.

40. Ibid, p. 259.

41. Ibid, p. 255.

42. Ibid, p. 311.

43. Ibid, p. 314 .

44. One could proceed without this restriction by bumping the original category up to the Heyting algebra of all cribles on $C$.

45. Smith and McIntyre, op. cit., pp. 256-258.

46. I would like to thank Dr. V. Huber-Dyson for the years that she spent teaching me Logic and Category Theory, Dr. H. Korte for encouraging this particular line of investigation and Dr. P. Engstrom for reading and commenting on two drafts of this paper. (Any misconceptions or shortcomings are the fault of the author and not of the above mentioned persons.) I also appreciated the comments made by two anonymous readers as well as the editor of this journal. In addition I would like to thank the Faculty of Graduate Studies and Research at the University of Regina for financial support which made this research possible, and King's College, University of Western Ontario, for a research grant that was used in the preparation of the final draft of this manuscript. 